Hans P. Heinig, Department of Mathematics, McMaster University, Hamilton, Ontario, Canada L8S 4K1

Lech Maligranda, Department of Mathematics, Lulea University, S-95187 Lulea, Sweden.

\title{
Chebyshev Inequality in Function Spaces ${ }^{1}$
}

We study the classical Chebyshev inequality

$$
\int_{0}^{a} x(s) d s \int_{0}^{a} y(s) d s \leq a \int_{0}^{a} x(s) y(s) d s
$$

and prove new variants, generalizations and abstractions. Among these are Chebyshev's inequality for strongly increasing functions, positive convex and concave functions, and generalizations of the Ky Fan inequality. Our abstractions involve Chebyshev's inequality in Banach function spaces and symmetric spaces. These considerations lead to generalizations of inequalities of Favard and Barnes. The constants that arise are studied and shown to be sharp in general.

\section{Introduction.}

Let $x$ and $y$ be positive decreasing functions on $I=[0, a], a>0$, then Chebyshev's inequality asserts that the product of the integral averages of $x$ and $y$ is not larger then the integral average of $x y$. That is,

$$
\int_{0}^{a} x(s) d s \int_{0}^{a} y(s) d s \leq a \int_{0}^{a} x(s) y(s) d s .
$$

Since its first proof in 1882 the inequality has been studied extensively and numerous variants, generalizations and extensions appeared in the literature. For example different proofs were given - we shall give five in the next

\footnotetext{
${ }^{1}$ AMS subject classification (1985): Primary 26D15, Secondary 46 E30
}

Key words and phrases: Chebysev inequality, Banach function spaces, symmetric spaces, Ky Fan inequality 
section, - the monotonicity conditions of the functions were replaced by concavity and convexity and the constant arising from these generalizations has, in many cases, been shown to be best possible. Other generalizations result by introducing weight functions or replace Lebesgue measure by general measures. Then the question is to find all functions and measures for which Chebyshev's inequality holds ([14]).

In this paper we give a number of new variants, generalizations and abstractions of Chebyshev's inequality.

The next section contains several proofs of the inequality, remarks and observations, and a conditional characterization of functions for which the inequality holds. A proof of Chebyshev's inequality (with different constant) is also given for strongly increasing functions (THEOREM 2.8) and the extension to products of functions and convex functions is deduced (COR. 2.10).

In terms of Lebesgue spaces, Chebyshev's inequality is

$$
\|x\|_{L_{1}(0, a)}\|y\|_{L_{1}(0, a)} \leq a\|x y\|_{L_{1}(0, a)} .
$$

This suggests that the inequality has an analogue in general Banach function spaces. That is, the $L_{1}$-norm in (1.1) can be replaced by Banach function norms or the norm of symmetric spaces. Of course the endpoint a of the interval $I=[0, a]$ in (1.1) will then have a different form. These abstractions and generalizations are contained in Section 3. In order to keep the paper self-contained various definitions and results (some with proofs) needed in the sequel are given here also. THEOREM 3.10 contains Chebyshev's inequality in Banach function spaces with constant 2. It is shown that in general the constant is sharp, although for symmetric spaces with Fatou property we show that the constant is 1 (THEOREM 3.13).

These abstractions - via a lemma - lead to generalizations of inequalities of Favard and Barnes and in addition provide new proofs of Grüss' form of Chebyshev's inequality for concave functions. These results with some abstract formulations of the inequality for concave functions in symmetric spaces are contained in Section 4.

The inequality of $\mathrm{Ky}$ Fan has the form

$$
\int_{0}^{a} \int_{0}^{a} K(s, t) x(s) y(t) d s d t \leq B \int_{0}^{a} x(s) y(s) d s
$$

where $K(s, t)$ is integrable over $I$ in $s$ and in $t$ with bound $B$ and $x, y$ are decreasing functions. In Section 5 we weaken the conditions imposed on 
$K$ and replace $x(s) y(t)$ by $f(s, t)$, where $f$ is decreasing in each variable separately. The inequality corresponding to (1.2) has then constant $2 B$ which is shown to be sharp (THEOREM 5.2). However, we also prove that for a large class of functions $f$, narnely the positive set functions the corresponding inequality has constant $B$. We conclude with some brief comments on P.L. Chebyshev's professional life.

Throughout we shall write increasing (function) instead of non-decreasing and decreasing shall mean non-increasing. Similarly, positive (negative) is to be interpreted as non-negative (non-positive). The interval $I$ will be the closed interval $I=[0, a]$. In a few instances, however, we write $[0, a)$ and $(0, a)$. We also denote by $1_{E}$ the characteristic function of the set $E$.

\section{Chebyshev's inequality, proofs and classi- cal generalizations.}

We give a number of proofs of Chebyshev's inequality and a new proof of a conditional characterization of those functions for which the inequality holds. In addition we prove the inequality for strongly increasing functions.

Theorem 2.1 (Chebyshev). Let $x$ and $y$ be positive decreasing functions on $I=[0, a]$ then

$$
\int_{0}^{a} x(s) d s \int_{0}^{a} y(s) d s \leq a \int_{0}^{a} x(s) y(s) d s .
$$

First proof. Since the hypotheses imply

$$
[x(s)-x(t)][y(s)-y(t)] \geq 0, \quad s, t \varepsilon I
$$

we obtain

$$
\begin{aligned}
0 & \leq \int_{0}^{a} \int_{0}^{a}[x(s)-x(t)][y(s)-y(t)] d t d s \\
& =\int_{0}^{a} \int_{0}^{a}[x(s) y(s)-x(s) y(t)-x(t) y(s)+x(t) y(t)] d t d s \\
& =\int_{0}^{a}\left[a x(s) y(s)-x(s) \int_{0}^{a} y(t) d t-y(s) \int_{0}^{a} x(t) d t+\int_{0}^{a} x(t) y(t) d t\right] d s \\
& =2\left[a \int_{0}^{a} x(s) y(s) d s-\int_{0}^{a} x(s) d s \int_{0}^{a} y(s) d s\right] .
\end{aligned}
$$


Thus (2.1) follows.

Second proof (when either $x$ or $y$ is continuous). Let $x$ be continuous. Then by the Mean Value Theorem there is a $c \varepsilon(0, a)$ such that

$$
\int_{0}^{a} x(s) d s=x(c) a \quad \text { or } \quad \int_{0}^{a}[x(s)-x(c)] d s=0 .
$$

Therefore

$$
\int_{0}^{a}[x(s)-x(c)] y(s) d s=\int_{0}^{a}[x(s)-x(c)][y(s)-y(c)] d s
$$

and so

$$
\begin{aligned}
\int_{0}^{a} x(s) d s \int_{0}^{a} y(s) d s & =a \int_{0}^{a} x(c) y(s) d s \\
& =a \int_{0}^{a} x(s) y(s) d s-a \int_{0}^{a}[x(s)-x(c)][y(s)-y(c)] d s .
\end{aligned}
$$

But by assumption $[x(s)-x(c)][y(s)-y(c)] \geq 0$ and so (2.1) follows.

Third proof (Sapogov [23]). For $t \varepsilon I$, define

$$
F(t)=x(t)-\frac{1}{a} \int_{0}^{a} x(s) d s .
$$

Clearly $F$ is decreasing on $I, F(0) \geq 0$ and $F(a) \leq 0$. Therefore, there exists a $c \varepsilon I$ such that $F(t) \geq 0$ for $t \varepsilon[0, c)$ and $F(t) \leq 0$ for $t \varepsilon(c, a]$. Hence

$$
\begin{aligned}
\int_{0}^{a}\left[x(t)-\frac{1}{a} \int_{0}^{a} x(s) d s\right] y(t) d t & =\int_{0}^{a} F(t) y(t) d t \\
& =\int_{0}^{c} F(t) y(t) d t+\int_{c}^{a} F(t) y(t) d t \\
& \geq y(c) \int_{0}^{c} F(t) d t+y(c) \int_{c}^{a} F(t) d t \\
& =y(c) \int_{0}^{a} F(t) d t \\
& =y(c) \int_{0}^{a}\left[x(t)-\frac{1}{a} \int_{0}^{a} x(s) d s\right] d t=0
\end{aligned}
$$

and (2.1) follows.

Fourth proof (Stein [28]). Since $x$ is decreasing, $x$ can be approximated by a sum of a finite number of step functions that have a single jump. It 
suffices then to establish (2.1) in the special case when $x(s)=1$ for $0 \leq s \leq c$ and $x(s)=0$ if $c<s \leq a$. But $y$ is decreasing, so

$$
\begin{aligned}
\int_{0}^{a} x(s) d s \int_{0}^{a} y(s) d s & =c \int_{0}^{a} y(s) d s \leq c \int_{0}^{c} y(s) d s+c y(c)(a-c) \\
& \leq c \int_{0}^{c} y(s) d s+(a-c) \int_{0}^{c} y(s) d s \\
& =a \int_{0}^{c} y(s) d s=a \int_{0}^{a} x(s) y(s) d s
\end{aligned}
$$

and again the result follows (Note that we have shown here that integral averages of decreasing functions are decreasing as the upper terminal increases).

Fifth proof. For $t \varepsilon I$ define $f$ by

$$
f(t)=t \int_{0}^{t} x(s) y(s) d s-\int_{0}^{t} x(s) d s \int_{0}^{t} y(s) d s .
$$

Clearly $f$ is continuous and differentiable a.e. anc

$$
\begin{aligned}
f^{\prime}(t) & =\int_{0}^{t} x(s) y(s) d s+t x(t) y(t)-x(t) \int_{0}^{t} y(s) d s-y(t) \int_{0}^{t} x(s) d s \\
& =\int_{0}^{t}[x(s)-x(t)][y(s)-y(t)] d s \geq 0
\end{aligned}
$$

by (2.2). Hence $f$ is increasing on $I$ and so $f(a) \geq f(0)=0$. But that is again (2.1).

Remark 2.2 i) The first proof is due to K.A. Andreiev (1883) and A.N. Korkin (1883). However, many authors ascribe the proof to F. Franklin (1885). The second proof is due to T. J. Stieltjes (1891), N. Ja. Sonin (1898) and H. Brunn (1903). It was also published by O. Dunkel (1924). For more about the history and priority of Chebyshev's inequality we refer to [21].

ii) If $x=y$ on $I$, then (2.1) holds (without restrictions) by Hölder's inequality.

iii) If $w$ is a positive integrable function on $I$ and $v: I \rightarrow I$ is measurable (c.f. [28]) then

$$
\int_{0}^{a} x(v(s)) w(s) d s \int_{0}^{a} y(v(s)) w(s) d s \leq \int_{0}^{a} w(s) d s \int_{0}^{a} x(v(s)) y(v(s)) w(s) d s
$$


whenever $x$ and $y$ are positive and decreasing on $I$. Indeed in terms of measures $\mu$, the inequality

$$
\int_{0}^{a} x(s) d \mu(s) \int_{0}^{a} y(s) d \mu(s) \leq \int_{0}^{a} d \mu(s) \int_{0}^{a} x(s) y(s) d \mu(s)
$$

holds for all $x$ and $y$ satisfying (2.2) if and only if $\mu$ is a positive measure. Moreover (2.3) holds for all positive measures if and only if $x$ and $y$ satisfy (2.2) $([14])$.

iv) It is obvious from the proof that Chebyshev's inequality holds whenever $x$ and $y$ satisfy (2.2). We say that positive functions $x$ and $y$ are similarly ordered (S.O.) on $I$ if (2.2) is satisfied.

Corollary 2.3 If $x$ and $y$ are S.O. on I then (2.1) holds.

Of course if $x$ and $y$ are both increasing or both decreasing then they are S.O. If $x$ is positive on $I$ and $c+\inf \{x(s): s \varepsilon I\} \geq 0$ then the pairs $x, c+x$ and $x^{2}, x$ are S.O. Also since

$[x(s)+y(s)-x(t)-y(t)][y(s)-y(t)]=[x(s)-x(t)][y(s)-y(t)]+[y(s)-y(t)]^{2}$ and

$[x(s) y(s)-x(t) y(t)][y(s)-y(t)]=x(s)[y(s)-x(t)]^{2}+y(t)[x(s)-y(t)][y(s)-y(t)]$ it follows that $x+y, y$ and $x y, y$ are S.O. whenever $x, y$ are.

One might conjecture that S.O. on $I$ is necessary and sufficient for Chebyshev's inequality. Note however, that the second proof of THEOREM 2.1 required only that the product $[x(s)-x(c)][y(s)-y(c)] \geq 0$, where $x$ is continuous in $I$ and $c$ is given by

$$
\int_{0}^{a} x(s) d s=a x(c)
$$

It is not difficult to give an example of functions $x$ and $y$ satisfying these conditions without being S.O. on $I$, yet Chebyshev's inequality holds.

Example 2.4 Let $a=1, x(s)=2-s$ and $y(s)=1+s-2 s^{2}$. Then $c=\frac{1}{2}$ and $[x(s)-x(c)][y(s)-y(c)]=\frac{1}{2} s(1-2 s)^{2} \geq 0$. Clearly $x$ decreases on $[0,1]$ while $y$ increases on $\left[0, \frac{1}{4}\right]$ and decreases on $\left[\frac{1}{4}, 1\right]$. Moreover, if $s \varepsilon\left[0, \frac{1}{4}\right)$, $\left[x(s)-x\left(\frac{1}{4}\right)\right]\left[y(s)-y\left(\frac{1}{4}\right)\right]<0$, so that $x, y$ are not S.O. while

$$
\int_{0}^{1} x(s) d s \int_{0}^{1} y(s) d s=\frac{3}{2} \cdot \frac{5}{6}<\frac{4}{3}=\int_{0}^{1} x(y) y(s) d s .
$$


We now consider the averaging operator of $x$ :

$$
(A x)(t)=\frac{1}{t} \int_{0}^{t} x(s) d s .
$$

If $x$ is positive and decreasing on $(0, a]$ so is $A x$, although the converse fails in general. Now if $x$ and $y$ are decreasing on $(0, a]$ then by (2.1) with $t \leq a$, $(A x)(t)(A y)(t) \leq(A x y)(t)$ and since $A x$ and $A y$ are decreasing it follows also from (2.1) that

$$
\begin{aligned}
{\left[\frac{1}{a} \int_{0}^{a}(A x)(t) d t\right]\left[\frac{1}{a} \int_{0}^{a}(A y)(t) d t\right] } & \leq \frac{1}{a} \int_{0}^{a}(A x)(t)(A y)(t) d t \\
& \leq \frac{1}{a} \int_{0}^{a}(A x y)(t) d t
\end{aligned}
$$

But

$$
\int_{0}^{a}(A x)(t) d t=\int_{0}^{a} t^{-1} \int_{0}^{t} x(s) d s d t=\int_{0}^{a} x(s) \int_{s}^{a} t^{-1} d t d s=\int_{0}^{a} x(s)\left(\ln \frac{a}{s}\right) d s
$$

and similarly for other integrals. Therefore the previous inequality has the form

$$
\left[\frac{1}{a} \int_{0}^{a} x(s)\left(\ln \frac{a}{s}\right) d s\right]\left[\frac{1}{a} \int_{0}^{a} y(s)\left(\ln \frac{a}{s}\right) d s\right] \leq \frac{1}{a} \int_{0}^{a} x(s) y(s) \ln \left(\frac{a}{s}\right) d s .
$$

Since the argument carries over to second and higher averages we obtain on iterating

Proposition 2.5 If $x$ and $y$ are positive and decreasing on $I$ then for $n \varepsilon N$

$$
\int_{0}^{a} x(s)\left(\ln \frac{a}{s}\right)^{n} d s \int_{0}^{a} y(s)\left(\ln \frac{a}{s}\right)^{n} d s \leq a n ! \int_{0}^{a} x(s) y(s)\left(\ln \frac{a}{s}\right)^{n} d s .
$$

The next result due to Steffensen [27] is a conditional characterization of the functions for which Chebyshev's inequality holds.

Theorem 2.6 Let $x$ be a positive decreasing function on I. If $y$ is positive and $A y(a)>0$, then (2.1) holds if and only if $(A y)(a) \leq(A y)(t)$ for all $t \varepsilon(0, a)$.

Note that the function $y$ of EXAMPLE 2.4 satisfies this condition (with $a=1$ ) while $A y$ is increasing on $\left(0, \frac{3}{8}\right)$ and decreasing on $\left[\frac{3}{8}, 1\right]$.

We shall give another proof of THEOREM 2.6, but for it we require the following known Hardy type lemma: 
Lemma 2.7 Let $f_{1}$ and $f_{2}$ be positive integrable functions on $I$ and suppose that for $t \varepsilon I, \int_{0}^{t} f_{1}(s) d s \leq \int_{0}^{t} f_{2}(s) d s$. If $g$ is positive and decreasing on $I$, then

$$
\int_{0}^{a} f_{1}(s) g(s) d s \leq \int_{0}^{a} f_{2}(s) g(s) d s
$$

Proof. Let $f(s)=f_{2}(s)-f_{1}(s)$ and $F(t)=\int_{0}^{t} f(s) d s$. Then for $0 \leq t \leq a$, $F(t) \geq 0$ and $F(0)=0$. If $g(0+)<\infty$, integration by parts yields

$$
\int_{0}^{a} f(t) g(t) d t=\int_{0}^{a} g(t) d F(t)=g(a) F(a)-\int_{0}^{a} F(t) d g(t) \geq 0
$$

and the result follows.

If $g(0+)=\infty$, define $g_{\alpha}(s)=g(\alpha)$ if $s \varepsilon[0, \alpha]$ and equal to $g(s)$ if $s \varepsilon(\alpha, a]$. Clearly $g_{\alpha}(s) \uparrow g(s)$ as $\alpha \rightarrow 0$ and by what we have shown

$$
\int_{0}^{a} f_{1}(s) g_{\alpha}(s) d s \leq \int_{0}^{a} f_{2}(s) g_{\alpha}(s) d s \leq \int_{0}^{a} f_{2}(s) g(s) d s .
$$

Hence by Fatou's lemma

$$
\int_{0}^{a} f_{1}(s) g(s) d s \leq_{\alpha} \varliminf_{\alpha \rightarrow 0+} \int_{0}^{a} f_{1}(s) g_{\alpha}(s) d s \leq \int_{0}^{a} f_{2}(s) g(s) d s .
$$

Note that if in LEMMA $2.7 \mathrm{~g}$ is positive and increasing on $I$ and in addition

$$
\int_{0}^{a} f_{1}(s) d s=\int_{0}^{a} f_{2}(s) d s, \text { then } \int_{0}^{a} f_{1}(s) g(s) d s \geq \int_{0}^{a} f_{2}(s) g(s) d s .
$$

We are now in a position to prove THEOREM 2.6.

Proof. Let $f_{1}(s)=1, f_{2}(s)=a y(s) / \int_{0}^{a} y(u) d u$, then for $t \varepsilon I$

$$
\int_{0}^{t} f_{1}(s) d s=t \leq t(A y)(t) /(A y)(a)=a \int_{0}^{t} y(u) d u / \int_{0}^{a} y(u) d u=\int_{0}^{a} f_{2}(s) d s
$$

and hence by LEMMA 2.7

$$
\int_{0}^{a} x(s) d s \leq \int_{0}^{a} x(s) f_{2}(s) d s .
$$

But this is clearly equivalent to (2.1).

The converse follows on taking $x(s)=1_{[0, t]}(s), t \varepsilon I$, in (2.1). 
In a similar way one shows that for decreasing $x$ on $I$

$$
\int_{0}^{a} x(s) w(s) d s \int_{0}^{a} y(s) w(s) d s \leq \int_{0}^{a} w(s) d s \int_{0}^{a} x(s) y(s) w(s) d s,
$$

where $w$ is positive and integrable on $I$, holds if and only if $\left(A_{w} y\right)(a) \leq$ $\left(A_{w} y\right)(t), t \varepsilon[0, a)$. Here $\left(A_{w} y\right)(t)=\int_{0}^{t} y(s) w(s) d s / \int_{0}^{t} w(s) d s$. For this result one defines $f_{1}=w$ and

$$
f_{2}(s)=y(s) w(s) \int_{0}^{a} w(u) d u / \int_{0}^{a} y(u) w(u) d u .
$$

We noted that THEOREM 2.1 holds also for increasing $x$ and $y$ on $I$. If one strengthens this condition to strongly increasing (i.e. $z(s)$ and $s^{-\gamma} z(s)$, are increasing for some $\gamma>0$ ) the following result holds:

Theorem 2.8 Let $x$ and $y$ be positive increasing functions on I such that $s^{-\alpha} x(s)$ and $s^{-\beta} y(s)$ are increasing for some $\alpha \geq 0$ and $\beta \geq 0$. Then

$$
\int_{0}^{a} x(s) d s \int_{0}^{a} y(s) d s \leq \frac{\alpha+\beta+1}{(\alpha+1)(\beta+1)} a \int_{0}^{a} x(s) y(s) d s .
$$

If $x(s)=C_{1} s^{\alpha}, y(s)=C_{2} s^{\beta}, C_{1}, C_{2}>0$ then equality in (2.4) is attained.

Proof. Without loss of generality assume $\alpha \leq \beta$. Since $x_{0}(s)=s^{-\alpha} x(s)$, $y_{0}(s)=s^{-\beta} y(s)$ are increasing then by Chebyshev's inequality with weights (c.f. REMARK 2.2 iii))

$$
\begin{aligned}
\int_{0}^{a} x(s) d s \int_{0}^{a} y(s) d s & =\int_{0}^{a} x_{0}(s) s^{\alpha} d s \int_{0}^{a} y_{0}(s) s^{\beta-\alpha} s^{\alpha} d s \\
& \leq \int_{0}^{a} s^{\alpha} d s \int_{0}^{a} x_{0}(s) y_{0}(s) s^{\beta-\alpha} s^{\alpha} d s \\
& =\frac{a^{\alpha+1}}{\alpha+1} \int_{0}^{a} x_{0}(s) y_{0}(s) s^{\beta} d s
\end{aligned}
$$

But $s^{\alpha}$ and $x_{0}(s) y_{0}(s)$ are also increasing, so again by Chebyshev's inequality with weight, we obtain

$$
\begin{aligned}
\int_{0}^{a} s^{\alpha} s^{\beta} d s \int_{0}^{a} x_{0}(s) y_{0}(s) s^{\beta} d s & \leq \int_{0}^{a} s^{\beta} d s \int_{0}^{a} x_{0}(s) y_{0}(s) s^{\alpha} s^{\beta} d s \\
& =\frac{a^{\beta+1}}{\beta+1} \int_{0}^{a} x(s) y(s) d s .
\end{aligned}
$$

This together with the previous inequality yields (2.4). 
Remark 2.9 i) It is not difficult to see that a corresponding result holds also for decreasing $x$ and $y$ with the property that $s^{\alpha} x(s)$ and $s^{\beta} y(s), \alpha \geq 0$, $\beta \geq 0$ are decreasing and $0<\alpha+\beta<1$.

ii) Applying induction on THEOREM 2.8 yields a more general result: If $x_{1}, x_{2}, \ldots, x_{n}$ are positive increasing functions on I such that $s^{-\alpha_{k}} x(s), \alpha_{k} \geq$
$0, k=1,2, \ldots, n$ are increasing on $(0, a)$, then

$$
\prod_{k=1}^{n} \int_{0}^{a} x_{k}(s) d s \leq \frac{1+\sum_{k=1}^{n} \alpha_{k}}{\prod_{k=1}^{n}\left(1+\alpha_{k}\right)} a^{n-1} \int_{0}^{a} \prod_{k=1}^{n} x_{k}(s) d s
$$
As a consequence of this result obtain the following Theorem of Andersson
[2]: Corollary 2.10 If $x_{1}, x_{2}, \ldots, x_{n}$ are positive convex functions on $I$ and $x_{k}(0)=$
$0, k=1,2, \ldots, n$, then

$$
\prod_{k=1}^{n} \int_{0}^{a} x_{k}(s) d s \leq \frac{n+1}{2^{n}} a^{n-1} \int_{0}^{a} \prod_{k=1}^{n} x_{k}(s) d s .
$$

The result follows at once if we show that $s^{-1} x_{k}(s), k=1,2, \ldots, n$ are increasing on $(0, a)$. But if $0<s<t$, this follows from the convexity of $x_{k}$ :

$$
s^{-1} x_{k}(s)=s^{-1} x_{k}\left(\frac{s}{t} t+\left(1-\frac{s}{t}\right) 0\right) \leq t^{-1} x_{k}(t) .
$$

We conclude this section with another application of Chebyshev's inequality.

It is clear that if $0 \leq x \varepsilon L_{q}([0, a])$ and $1 \leq p \leq q$ then Hölder's inequality implies $\|x\|_{p} \leq a^{1 / p-1 / q}\|x\|_{q}$. If however we know additionally that $x_{0}(s)=s^{-\alpha} x(s), \alpha>0$ is increasing on $(0, a)$ then by Jensen's, respectively, Chebyshev's inequality (REMARK 2.2 iii))

respectively

$$
\left(\frac{\int_{0}^{a} x(s)^{p} d s}{\int_{0}^{a} s^{\alpha p} d s}\right)^{q / p}=\left(\frac{\int_{0}^{a} x_{0}(s)^{p} s^{\alpha p} d s}{\int_{0}^{a} s^{\alpha p} d s}\right)^{q / p} \leq \frac{\int_{0}^{a} x_{0}(s)^{q} s^{\alpha p} d s}{\int_{0}^{a} s^{\alpha p} d s}
$$

$$
\begin{aligned}
\int_{0}^{a} x_{0}(s)^{p} s^{\alpha p} d s \int_{0}^{a} s^{\alpha(q-p)} s^{\alpha p} d s & \leq \int_{0}^{a} s^{\alpha p} d s \int_{0}^{a} x_{0}(s)^{q} s^{\alpha(q-p)} s^{\alpha p} d s \\
& =\int_{0}^{a} s^{\alpha p} d s \int_{0}^{a} x(s)^{q} d s
\end{aligned}
$$


from which the following sharper inequality is obtained: If $\alpha>0, s^{-\alpha} x(s)$ is increasing in $[0, a]$ and $1 \leq p \leq q$, then

$$
\left(\frac{\alpha p+1}{a}\right)^{1 / p}\|x\|_{p} \leq\left(\frac{\alpha q+1}{a}\right)^{1 / q}\|x\|_{q}
$$

\section{Chebyshev's inequality in Banach function spaces.}

In this section we define Banach function spaces and symmetric space and give sorne of their fundamental properties. While most of the results may be found in the literature ([6], [17], [29]), we present them here to make the paper self contained and for easy reference when the Chebyshev inequality is proved in this abstract setting. We begin with some definitions.

$L^{0}(I)$ denotes the space of all (equivalence classes of) real valued measurable functions on I with Lebesgue measure. This space is a complete topological vector space with convergence in measure as a topology. $A B G$ nach subspace $X=\left(X,\|.\|_{X}\right)$ of $L^{0}(I)$ is called a Banach function space on $I$ or simply Banach function space if it has the ideal property:

(I) $x \varepsilon L^{0}(I), y \varepsilon X$ and $|x| \leq|y|$ a.e. on $I$ implies $x \varepsilon X$ and $\|x\| x \leq\|y\|_{X}$.

It follows from this definition that $\|x\|_{X}=\|x\|_{X}$ for all $x \varepsilon X$. Moreover, the embedding of $X$ into $L^{0}(I)$ is continuous.

The Banach function space $X$, or more precisely the norm $\|\cdot\|_{X}$ :

a) is order continuous if $0 \leq x_{n} \downarrow 0$ a.e. implies $\left\|x_{n}\right\|_{X} \rightarrow 0$ as $n \rightarrow \infty$. $x \varepsilon X$.

b) has the weak Fatou property if $0 \leq x_{n} \uparrow x$ and $\sup \left\|x_{n}\right\|_{X}<\infty$ implies

c) has the Fatou property if it has the weak Fatou and satisfies $\left\|x_{n}\right\|_{X} \rightarrow$ $\|x\|$ as $n \rightarrow \infty$.

If $x \in X$, then $x$ vanishes a.e. on $I_{1}:=I \backslash \operatorname{supp} X$, where $\operatorname{supp} X=$ $\sup \left\{1_{\operatorname{supp}} x: x \varepsilon X\right\}$. Thus for our purposes we simply remove $I_{1}$ from $I$ and suppose that $I=\operatorname{supp} X$.

The Köthe dual or associate space $X^{\prime}$ of a Banach function space $X$ on $I$ is the space of all $y \varepsilon L^{0}(I)$ for which $x y \varepsilon L_{1}$ for each $x \varepsilon X$. For $y \varepsilon X^{\prime}$ we 
define

$$
\|y\|_{X^{\prime}}=\sup \left\{\int_{I}|x y| d m: x \in X \text { and }\|x\|_{X} \leq 1\right\} .
$$

where $m$ denotes Lebesgue measure.

To insure that this is a norm rather than a semi-norm we shall assume, as indicated above, that $I=\operatorname{supp} X$. However, always supp $X^{\prime}=\operatorname{supp} X$. It is clear that $X^{\prime}$ has the Fatou property (since the integral and supremum has) and that Hölder's inequality holds: If $x \in X$ and $y \varepsilon X^{\prime}$, then $x y$ is integrable and

$$
\int_{I}|x y| d m \leq\|x\|_{X}\|y\|_{X^{\prime}}
$$

It follows from (3.1) that the associate space $X^{\prime}$ is a subspace of the conjugate space $X^{*}$. These spaces are identical if and only if $X$ is order continuous. The second associate space is defined by $X^{\prime \prime}=\left(X^{\prime}\right)^{\prime}$. It is clear that $X$ can be embedded in $X^{\prime \prime}$ in a natural way and for all $x \varepsilon X,\|x\|_{X^{\prime \prime}} \leq\|x\|_{X}$. The following theorem of Lorentz and Luxemburg $([6$, THM. 2.7] or [29,
THM. 4, p. 472]) is basic:

Theorem 3.1 $X^{\prime \prime}=X$ isometrically if and only if $X$ has the Fatou property. This yields at once

Corollary 3.2 If a Banach function space $X$ is either order continuous (since $X^{\prime}=X^{*}$ ) or $X$ has the Fatou property, then for $x \varepsilon X$

$$
\|x\|_{X}=\sup \left\{\int_{I}|x y| d m: y \varepsilon X^{\prime},\|y\|_{X^{\prime}}=1\right\} .
$$

Theorem 3.3 (Amemiya [1]). If $X$ has the weak Fatou property, then there is a constant $j, 0<j \leq 1$, such that $0 \leq x_{n} \rightarrow x, n \rightarrow \infty$, implies
$j\|x\|_{X} \leq \liminf _{n \rightarrow \infty}\left\|x_{n}\right\|_{X}$.

Proof. As in [1] we prove first that if $0 \leq x_{n} \uparrow x, n \rightarrow \infty$, then for some $j \varepsilon(0,1], j\|x\|_{X} \leq \sup _{n}\left\|x_{n}\right\|_{X}$. If such $j$ cannot be found, then there exists $x_{m} \geq 0$ and a sequence $0 \leq x_{m n} \uparrow x_{m}$, such that $\left\|x_{m n}\right\|_{X} \leq 1$ and
$\left\|x_{m}\right\|_{X} \geq m^{3}$ for $m=1,2, \ldots$, . Now let

$$
y_{n}(s)=\sum_{m=1}^{n} m^{-2} x_{m n}(s)
$$


then $y_{n} \varepsilon X, y_{n+1} \geq y_{n}$ and $\left\|y_{n}\right\|_{X} \leq \sum_{m=1}^{\infty} m^{-2}$ for all $n$. Hence $\sup _{n} y_{n} \varepsilon X$ by the weak Fatou property.

On the other hand, for $n \geq N, y_{n}(s) \geq \sum_{n=1}^{N} m^{-2} x_{m n}(s)$ and since $x_{m n} \nearrow x_{m}$, it follows that $\sup _{n} y_{n}(s) \geq \sum_{n=1}^{N} m^{-2} x_{m}(s)$. This implies that $\left\|\sup y_{n}\right\|_{X} \geq N^{-2}\left\|x_{N}\right\|_{X} \geq N$, for any $N$ in contradiction to $\sup _{n} y_{n} \varepsilon X$.

Now, if $0 \leq x_{n} \rightarrow x, n \rightarrow \infty$, let $z_{n}=\inf \left\{x_{n}, x_{n+1}, \ldots\right\}$, then $z_{n} \uparrow x$ as $n \rightarrow \infty$ and so $j\|x\|_{X} \leq \sup _{n}\left\|z_{n}\right\|_{X} \leq \liminf \left\|x_{n}\right\|_{X}$.

If $X$ is a Banach function space, we shall assume that $1_{I} \in X$. This is equivalent to the continuous embedding of $L_{\infty}$ into $X$. The assumption also guarantees that the fundamental function on $X$, that is, $\varphi_{X}(t)=\left\|1_{[0, t]}\right\|_{X}$, $t \varepsilon I$, is well defined. We note that $1_{I} \varepsilon X^{\prime}$ if and only if $X$ is continuously embedded in $L_{1}$.

Important classes of Banach function spaces are symmetric or rearrangement invariant spaces. To define these we need some definitions.

The distribution function $d_{x}$ of $x \varepsilon L^{0}(I)$ is defined by

$$
d_{x}(\lambda)=m\{s \varepsilon I:|x(s)|>\lambda\}
$$

and the decreasing rearrangement $x *$ of $x$ on $[0, \infty)$ is the inverse of $d_{x}$, or more precisely $x *(t)=\inf \left\{\lambda \geq 0: d_{x}(\lambda) \leq t\right\}$. Note that by (3.2), $d_{x}(\lambda) \leq m(I)=a$, so that $x *(t)=0$ for $t \geq a$. Therefore $x *$ may be regarded as a function defined on $I$.

Two functions $x, y \varepsilon L^{0}(I)$ are equimeasurable if they have the same distribution function, i.e. $d_{x}(\lambda)=d_{y}(\lambda)$, for all $\lambda \geq 0$.

Definition 3.4 A Banach function space $X=\left(X,\|\cdot\|_{X}\right)$ is said to be symmetric or rearrangement invariant if

(RI) $\quad x \varepsilon L^{0}(I), y \varepsilon X$ and $d_{x}=d_{y}$ implies $x \varepsilon X$ and $\|x\|_{X}=\|y\|_{X}$.

Since $x^{*}$ of $x$ is equimeasurable with $x$, it is clear that $X$ is symmetric if $\|x\|_{X}=\left\|x^{*}\right\|_{X}$. The conditions (I) and (RI) are equivalent to the single condition:

$$
x \varepsilon L^{\mathrm{G}}(I), y \varepsilon X \text { and } x^{*}(t) \leq y^{*}(t), t \varepsilon I \text { implies } x \varepsilon X \text { and }\|x\|_{X} \leq\|y\|_{X} .
$$

The next result shows that symmetric spaces are "intermediate" between the Lebesgue spaces $L_{\infty}$ and $L_{1}$. 
Theorem 3.5 ([6, THM. 6.6], [17, THM. 4.1]). If $X$ is a non-trivial symmetric space, then $L_{\infty} \subset X \subset L_{1}$, with continuous embeddings. Furthermore $\varphi_{X}(t) \varphi_{X^{\prime}}(t)=t, t \varepsilon I$.

The $L_{p}, 1 \leq p \leq \infty$ spaces, the Lorentz space $L_{p, q}, 1<p<\infty, 1 \leq$ $q \leq \infty$ and Orlicz $L_{M}$ spaces are examples of symmetric spaces with the Fatou property. A function $\varphi$ defined on $I$ is said to be quasi-concave if $\varphi$ is increasing on $I, \varphi(t)=0$ if and only if $t=0$ and $\varphi(t) / t$ is decreasing on $(0, \alpha]$. The Lorentz space $\wedge_{\varphi}$, respectively, Marcinkiewicz space $M_{\varphi}$ consists of all $x \varepsilon L^{0}(I)$ for which the functional

$$
\|x\|_{\wedge_{\varphi}}=\int_{0}^{a} x^{*}(s) d \varphi(s):=\varphi(0+)\|x\|_{\infty}+\int_{0}^{a} x^{*}(s) \varphi^{\prime}(s) d s
$$

respectively $\|x\|_{M_{\varphi}}=\sup _{0<t<a} \varphi(t)\left(\frac{1}{t} \int_{0}^{t} x^{*}(s) d s\right)$ is finite, where $\varphi$ is quasiconcave. These spaces are also symmetric with the Fatou property and $\left(\wedge_{\varphi}\right)^{\prime}=M_{\varphi_{*},}$, where $\varphi_{*}(t)=t / \varphi(t)$, isometrically (cf. [6, p.p. 69-72], [17, p.p. 107-114]). that

If $x, y \varepsilon L^{0}(I)$, then a well known result of Hardy and Littlewood shows

$$
\int_{I}|x y| d m \leq \int_{0}^{*} x^{*}(s) y^{*}(s) d s
$$

while conversely ([6, THMS. 2.6., 2.7], [17, p. 69])

$$
\int_{0}^{a} x^{*}(s) y^{*}(s) d s=\sup \left\{\int_{I}|x z| d m: z \varepsilon L^{0}(I), d_{z}=d_{y}\right\}
$$

holds. Indeed the supremum is attained. This leads to

Theorem 3.6 Let $X$ be a symmetric space on I. Then the associate space $X^{\prime}$ is symmetric. Moreover

$$
\|y\|_{X^{\prime}}=\sup \left\{\int_{0}^{a} x^{*}(s) y^{*}(s) d s:\|x\|_{X} \leq 1\right\}
$$

and

$$
\|x\|_{X^{\prime \prime}}=\sup \left\{\int_{0}^{a} x^{*}(s) y^{*}(s) d s:\|y\|_{X^{\prime}} \leq 1\right\}
$$


The special roles of the spaces $M_{\varphi}$ and $\wedge_{\varphi}$ is reflected in the following facts: If $X$ is symmetric with fundamental function $\varphi_{X}=\varphi$, then $\wedge_{\varphi} \subset \wedge_{\tilde{\varphi}} \subset X \subset$ $M_{\varphi}$, where $\tilde{\varphi}$ is the smallest concave majorant of $\varphi$ on $I$. Here inclusion is continuous ([6], [17]).

Also, as an immediate consequence of COROLLARY 3.2 and THEOREM 3.6 we obtain the following result of Semenov [26]:

Theorem 3.7 If a symmetric space $X$ is either order continuous or has the Fatou property, then $X=\cap \wedge_{\varphi_{y}}$, where $\varphi_{y}(s)=\int_{0}^{s} y^{*}(u) d u$, and the intersection is over $y \varepsilon X^{\prime}$ with $\|y\|_{X^{\prime}} \leq 1$, and $\|x\|_{X}=\sup \left\{\|x\|_{\Lambda_{\varphi_{y}}}: y \varepsilon X^{\prime},\|y\|_{X^{\prime}} \leq\right.$ $1\}$.

A symmetric space $X$ on $I$ is said to have the majorant property of

$$
\begin{gathered}
x \varepsilon L^{0}(I), y \in X \text { and } \int_{0}^{t} x^{*}(s) d s \leq \int_{0}^{t} y^{*}(s) d s, t \varepsilon I \\
\text { implies } x \in X \text { and }\|x\|_{X} \leq\|y\|_{X} .
\end{gathered}
$$

The majorant property arises in the problem of interpolation between $L_{1}$ and $L_{\infty}$. In fact - as shown by Calderón and Mitjagin [17] - the symmetric space $X$ is an exact interpolation space between $L_{1}$ and $L_{\infty}$ if and only if $X$ has the majorant property.

Proposition 3.8 The $L_{p}$-spaces, $1 \leq p \leq \infty$, Lorentz spaces $\wedge_{\varphi}$ and Marcinkiewicz spaces $M_{\varphi}$ have the majorant property.

Proof. We prove this for the $L_{p}$-spaces only since the proof for $\wedge_{\varphi}$ is similar and the case $M_{\varphi}$ is obvious.

If $p=1$ there is nothing to prove and if $p=\infty$, then

$$
x^{*}(t) \leq \frac{1}{t} \int_{0}^{t} x^{*}(s) d s \leq \frac{1}{t} \int_{0}^{t} y^{*}(s) d s \leq\|y\|_{\infty}
$$

for all teI. Hence $\|x\|_{\infty} \leq\|y\|_{\infty}$. that

If $1<p<\infty$, then, because $\left(x^{*}\right)^{p-1}$ is decreasing LEMMA 2.7 shows

$$
\int_{0}^{a} x^{*}(s)^{p} d s \leq \int_{0}^{a} y^{*}(s) x^{*}(s)^{p-1} d s .
$$

But for any positive numbers $a$ and $b, a^{p}-b^{p} \leq p a^{p-1}(a-b)$, so that

$$
\int_{0}^{a}\left[x^{*}(s)^{p}-y^{*}(s)^{p}\right] d s \leq p \int_{0}^{a}\left[x^{*}(s)^{p}-x^{*}(s)^{p-1} y^{*}(s)\right] d s \leq 0
$$


and therefore $\|x\|_{p} \leq\|y\|_{p}$.

From this and THEOREM 3.7 it is easy to deduce the following general result:

Theorem 3.9 If a symmetric space is either order continuous or has the Fatou property, then it has the majorant property.

After this discussion we can prove Chebyshev's inequality in function spaces. We have two proofs of the following:

Theorem 3.10 Let $X$ be a Banach function space on $I$, such that $1_{I} \varepsilon X$. If $x, y \varepsilon X$ are positive, decreasing and $x y \varepsilon X$, then

$$
\|x\|_{X}\|y\|_{X} \leq 2 \varphi_{X}(a)\|x y\|_{X} .
$$

First proof. If $s, t \varepsilon I$, then

$$
\begin{aligned}
x(s) y(t) & =x(s) y(t) 1_{[0, t]}(s)+x(s) y(t) 1_{(t, a]}(s) \\
\leq & x(s) y(s) 1_{[0, t]}(s)+x(t) y(t) 1_{(t, a]}(s) \\
& <(o) y(0) 1_{[s, a]}(i)+x(i) y(t) 1_{(t, a]}(s) \\
\leq & x(s) y(s) 1_{[0, a]}(t)+x(t) y(t) 1_{[0, a]}(s) .
\end{aligned}
$$

Hence

$$
\|x\|_{X} y(t) \leq\|x y\|_{X} 1_{[0, a]}(t)+x(t) y(t) \varphi_{X}(a)
$$

and so

$$
\|x\|_{X}\|y\|_{X} \leq \varphi_{X}(a)\|x y\|_{X}+\varphi_{X}(a)\|x y\|_{X}=2 \varphi_{X}(a)\|x y\|_{X}
$$

Second proof. (if $X$ has the Fatou property). For $t \varepsilon I$ and any $0 \leq z_{1} \varepsilon X^{\prime}$ with $\left\|z_{1}\right\|_{X^{\prime}} \leq 1$

$$
\begin{aligned}
y(t) \int_{0}^{t} x(s) z_{1}(s) d s & \leq y(t)\left\|x 1_{[0, t]}\right\|_{X \prime \prime} \\
& \leq y(t)\left\|x 1_{[0, t]}\right\|_{X} \leq\left\|x y 1_{[0, t]}\right\|_{X} .
\end{aligned}
$$

In the same way, for any $0 \leq z_{2} \varepsilon X^{\prime}$ with $\left\|z_{2}\right\|_{X^{\prime}} \leq 1$

$$
x(t) \int_{0}^{t} y(s) z_{2}(s) d s \leq\left\|x y 1_{[0, t]}\right\|_{X} .
$$


Thus

$$
\begin{aligned}
& \frac{d}{d t}\left[\left(\int_{0}^{t} x(s) z_{1}(s) d s\right)\left(\int_{0}^{t} y(s) z_{2}(s) d s\right)\right] \\
& \quad \leq x(t) z_{1}(t) \int_{0}^{t} y(s) z_{2}(s) d s+y(t) z_{2}(t) \int_{0}^{t} x(s) z_{1}(s) d s \\
& \quad \leq\left[z_{1}(t)+z_{2}(t)\right]\left\|x y 1_{[0, t]}\right\|_{X} .
\end{aligned}
$$

Integrating from 0 to $a$ and applying Hölder's inequality we obtain

$$
\begin{aligned}
\int_{0}^{a} x(s) z_{1}(s) d s \int_{0}^{a} y(s) z_{2}(s) d s & \leq \int_{0}^{a}\left[z_{1}(t)+z_{2}(t)\right]\left\|x y 1_{[0, t]}\right\|_{X} d t \\
& \leq\left\|x y 1_{I}\right\| \int_{0}^{a}\left[z_{1}(t)+z_{2}(t)\right] d t \\
& \leq\left\|x y 1_{I}\right\|_{X}\left[\varphi_{X}(a)\left\|z_{1}\right\|_{X^{\prime}}+\varphi_{X}(a)\left\|z_{2}\right\|_{X^{\prime}}\right] \\
& \leq 2 \varphi_{X}(a)\left\|x y 1_{I}\right\|_{X} .
\end{aligned}
$$

Now taking the supremum of $z_{1}$ with $\left\|z_{1}\right\|_{X^{\prime}} \leq 1$ and then over $z_{2}$ with $\left\|z_{2}\right\|_{X^{\prime}} \leq 1$, we obtain $\|x\| X^{\prime \prime}\|y\|_{X^{\prime \prime}} \leq 2 \varphi(a)\|x y\|_{X}$ and this yields the result.

herian $u .1$ i roi symmetric spaces this result with quite difterent proof was also obtained by Sedaev [25]. In this paper the author refers to an unpublished report of V.I. Dmitriev and A.A. Sedaev, "Some inequalities for symmetric spaces" Voronezh 1977, in which (3.3) was proved with constant 3 .

The next example shows that there is a symmetric space without Fatou property (but weak Fatou property) for which the constant 2 in (3.3) is sharp.

Example 3.12 Let $X=L_{\infty}([0,1])$ and for $m, n=1,2, \ldots$,

$$
\|x\|=\max \left\{\frac{1}{m}\|x\|_{\infty}+\left(1-\frac{1}{m}\right)\|x\|_{1}, n \int_{0}^{1 / n} x^{*}(s) d s\right\} .
$$

Then $X=(X,\|\cdot\|)$ is a symmetric space on $[0,1]$ and $\varphi_{X}(1)=1$. Let $x_{n}=1_{[0, n-1]}, y_{m n}=m 1_{\left[0, n^{-2}\right]}+1_{\left(n^{-2}, 1\right]}$, then $x_{n}, y_{m n}$ are positive decreasing functions and $\left\|x_{n}\right\|=1$. Since

$$
\left\|y_{m n}\right\|=\max \left\{1+\left(1-\frac{1}{m}\right)\left(\frac{m}{n^{2}}+1-\frac{1}{n^{2}}\right), \frac{m}{n}+n\left(\frac{1}{n}-\frac{1}{n^{2}}\right)\right\} \rightarrow 2-\frac{1}{m}
$$


as $n \rightarrow \infty$ and

$$
\left\|x_{n} y_{m n}\right\|=\max \left\{1+\left(1-\frac{1}{m}\right)\left(\frac{m}{n^{2}}+\frac{1}{n}-\frac{1}{n^{2}}\right), \frac{m}{n}+n\left(\frac{1}{n}-\frac{1}{n^{2}}\right)\right\} \rightarrow 1
$$

as $n \rightarrow \infty$ it follows that

$$
\frac{\left\|x_{n}\right\|\left\|y_{m n}\right\|}{\varphi(1)\left\|x_{n} y_{m n}\right\|} \rightarrow 2-\frac{1}{m} \text { as } n \rightarrow \infty .
$$

As $m \rightarrow \infty$, this shows that the constant 2 is best possible.

THEOREMS 3.5 and 3.7 permit us now to prove the main result of this section, namely that for symmetric spaces with Fatou property the constant in (3.3) is 1 instead of 2.

Theorem 3.13 Let $X$ be a symmetric space on $I$, which is either order continuous or has the Fatou property. If $x, y \in X$ are positive decreasing functions on $I$ and $x y \in X$, then

$$
\|x\|_{X}\|y\|_{X} \leq \varphi_{X}(a)\|x y\|_{X}
$$

Proof. Observe that by THEOREM 3.5

$$
\begin{aligned}
& \sup \left\{\varphi_{z}(a): z \varepsilon X^{\prime},\|z\|_{X^{\prime}}=1\right\} \\
& \quad=\sup \left\{\int_{0}^{a} z^{*}(s) d s: z \varepsilon X^{\prime},\|z\|_{X^{\prime}}=1\right\}=\varphi_{X}(a) .
\end{aligned}
$$

Let $z_{1}, z_{2} \varepsilon X^{\prime},\left\|z_{1}\right\|_{X^{\prime}}=\left\|z_{2}\right\|_{X^{\prime}}=1$ and $\varphi_{i}(t)=\int_{0}^{t} z_{i}^{*}(s) d s, i=1,2$. Then

$$
\begin{aligned}
0 & \int_{0}^{a} \int_{0}^{a}[x(s)-x(t)][y(s)-y(t)] d \varphi_{1}(s) d \varphi_{2}(t) \\
= & \int_{0}^{a}\left[\int_{0}^{a} x(s) y(s) d \varphi_{1}(s)-y(t) \int_{0}^{a} x(s) d \varphi_{1}(s)\right. \\
& \left.\quad-x(t) \int_{0}^{a} y(s) d \varphi_{1}(s)+x(t) y(t) \varphi_{1}(a)\right] d \varphi_{2}(t) \\
=\varphi_{2}(a) & \int_{0}^{a} x(s) y(s) d \varphi_{1}(s)-\int_{0}^{a} y(t) d \varphi_{2}(t) \int_{0}^{a} x(s) d \varphi_{1}(s) \\
& \quad-\int_{0}^{a} x(t) d \varphi_{2}(t) \int_{0}^{a} y(s) d \varphi_{1}(s)+\varphi_{1}(a) \int_{0}^{a} x(t) y(t) d \varphi_{2}(t)
\end{aligned}
$$


and therefore

$$
\begin{aligned}
\int_{0}^{a} x(s) d \varphi_{1}(s) & \int_{0}^{a} y(t) d \varphi_{2}(t)+\int_{0}^{a} x(t) d \varphi_{2}(t) \int_{0}^{a} y(s) d \varphi_{1}(s) \\
& \leq \varphi_{2}(a) \int_{0}^{a} x(s) y(s) d \varphi_{1}(s)+\varphi_{1}(a) \int_{0}^{a} x(t) y(t) d \varphi_{2}(t) \\
& =\varphi_{2}(a)\|x y\|_{\wedge_{\varphi_{1}}}+\varphi_{1}(a)\|x y\|_{\wedge_{\varphi_{2}}} \\
& \leq \varphi_{2}(a) \sup _{\left\|z_{1}\right\|_{X^{\prime} \leq 1}}\|x y\|_{\wedge_{\varphi_{1}}}+\varphi_{1}(a) \sup _{\left\|z_{2}\right\|_{X^{\prime} \leq 1}}\|x y\|_{\wedge_{\varphi_{2}}} \\
& =\varphi_{2}(a)\|x y\|_{X}+\varphi_{1}(a)\|x y\|_{X}
\end{aligned}
$$

where the last inequality follows from THEOREM 3.7. By the above observation the last sum is not greater than

$$
\left[\sup _{\left\|z_{2}\right\| x_{X^{\prime}=1}} \varphi_{2}(a)+\sup _{\left\|z_{1}\right\|_{X^{\prime}=1}} \varphi_{1}(a)\right]\|x y\|_{X}=2 \varphi_{X}(a)\|x y\|_{X} .
$$

Hence we have shown that $\|x\|_{\wedge_{\varphi_{1}}}\|y\|_{\wedge_{\varphi_{2}}}+\|x\|_{\wedge_{\varphi_{2}}}\|y\|_{\wedge_{\varphi_{1}}} \leq 2 \varphi_{X}(a)\|x y\|_{X}$. Taking the supremum over $z_{1} \varepsilon X^{\prime}$ with $\left\|z_{1}\right\|_{X^{\prime}}=1$ we obtain from THEOREM $3.7\|x\|_{X}\|y\|_{\wedge_{\varphi_{2}}}+\|x\|_{\wedge_{\varphi_{2}}}\|y\|_{X} \leq 2 \varphi_{X}(a)\|x y\|_{X}$ and then taking the supremum over $z_{2} \varepsilon X^{\prime}$ with $\left\|z_{2}\right\|_{X^{\prime}}=1$ we obtain in the same way $\|x\|_{X}\|y\|_{X}+\|x\|_{X}\|y\|_{X} \leq 2 \varphi_{X}(a)\|x y\|_{X}$. This proves the theorem.

\section{Chebyshev inequality for concave functions.}

In this section we discuss Chebyshev's inequality for concave functions. We prove a generalization of Favard's inequality (LEMMA 4.2) which together with Grüss' theorem (THEOREM 4.1) yields a number of Chebyshev type inequalities in the abstract function theoretic setting.

Recall that a function $x$ on $I$ is concave if for $s, t \varepsilon I, 0 \leq \alpha \leq 1$, the inequality $\alpha x(s)+(1-\alpha) x(t) \leq x(\alpha s+(1-\alpha) t)$ holds. If this inequality is reversed, $x$ is said to be convex.

We shall give two proofs of the following theorem of Grüss [15]:

Theorem 4.1 Let $x, y$ be positive concave integrable functions on $I$. Then

$$
\int_{0}^{a} x(s) d s \int_{0}^{a} y(s) d s \leq \frac{3}{2} a \int_{0}^{a} x(s) y(s) d s .
$$


The inequality (4.1) is sharp in the sense that for the concave functions $x(s)=C_{1} s, y(s)=C_{2}(a-s), C_{1}, C_{2}>0$ equality in (4.1) holds.

Before proving this result we give a generalization of Favard's inequality to symmetric spaces via the following lemma:

Lemma 4.2 Let $x$ be a positive concave function on $I$. Then $x^{*}$, the rearrangement of $x$, is also concave on $I$ and for $0 \leq t \leq a$

$$
\int_{0}^{t} x^{*}(s) d s \leq \frac{t}{a}\left(2-\frac{t}{a}\right) \int_{0}^{a} x^{*}(s) d s .
$$

Moreover, if $X$ is a symmetric space on $I$ with the majorant property, then for $x \in X$

$$
\|x\|_{X} \leq 2 C(X) \frac{1}{a} \int_{0}^{a} x(s) d s
$$

where $C(X)=a^{-1}\left\|x_{0}\right\|_{X}$ and $x_{0}(s)=s$.

Proof. Let $A_{x}(\lambda)=\{s \varepsilon I: x(s)>\lambda\}, \lambda \geq 0$, then $d_{x}(\lambda)=m\left(A_{x}(\lambda)\right)$. Suppose $x$ is concave and $0 \leq \lambda_{1}, \lambda_{2}<$ ess sup $x$, then $A_{x}(\lambda)$ is a convex subset of $I$ and for $0<\alpha<1, A_{x}\left(\alpha \lambda_{1}+(1-\alpha) \lambda_{2}\right) \supset \alpha A_{x}\left(\lambda_{1}\right)+(1-\alpha) A_{x}\left(\lambda_{2}\right)$. From this and Brunn's inequality for convex subsets of $\mathbf{R}$ we obtain $d_{x}\left(\alpha \lambda_{1}+\right.$ $\left.(1-\alpha) \lambda_{2}\right)=m\left[A_{x}\left(\alpha \lambda_{1}+(1-\alpha) \lambda_{2}\right)\right] \geq m\left[\alpha A_{x}\left(\lambda_{1}\right)+(1-\alpha) A_{x}\left(\lambda_{2}\right)\right] \geq$ $\alpha m\left[A_{x}\left(\lambda_{1}\right)\right]+(1-\alpha) m\left[A_{x}\left(\lambda_{2}\right)\right]=\alpha d_{x}\left(\lambda_{1}\right)+(1-\alpha) d_{x}\left(\lambda_{2}\right)$. Hence $d_{x}$ is concave on $[0$, ess sup $x]$.

Now for $0 \leq t_{1}, t_{2}<a$ and $\varepsilon_{1}, \varepsilon_{2}>0$, let $\lambda_{i}=x^{*}\left(t_{i}\right)-\varepsilon_{i}, i=1,2$. Then $d_{x}\left(\lambda_{i}\right)>t_{i}, i=1,2$ and

$$
d_{x}\left(\alpha \lambda_{1}+(1-\alpha) \lambda_{2}\right) \geq \alpha d_{x}\left(\lambda_{1}\right)+(1-\alpha) d_{x}\left(\lambda_{2}\right)>\alpha t_{1}+(1-\alpha) t_{2},
$$

that is, $x^{*}\left(\alpha t_{1}+(1-\alpha) t_{2}\right)>\alpha \lambda_{1}+(1-\alpha) \lambda_{2}=\alpha x^{*}\left(t_{1}\right)+(1-\alpha) x^{*}\left(t_{2}\right)-$ $\alpha \varepsilon_{1}-(1-\alpha) \varepsilon_{2}$.

Also $d_{x}\left(\alpha \lambda_{1}\right) \geq \alpha d_{x}\left(\lambda_{1}\right)+(1-\alpha) d_{x}(0)=\alpha d_{x}\left(\lambda_{1}\right)+(1-\alpha) a>\alpha t_{1}+$ $(1-\alpha) a$, so that $x^{*}\left(\alpha t_{1}+(1-\alpha) a\right)>\alpha \lambda_{1}=\alpha x^{*}\left(t_{1}\right)-\alpha \varepsilon_{1}=\alpha x^{*}\left(t_{1}\right)+(1-$ $\alpha) x^{*}(a)-\alpha \varepsilon_{1}$. Since $\varepsilon_{1} \varepsilon_{2}$ are arbitrary, it follows that $x^{*}$ is concave.

To prove (4.2) observe that

$$
t\left(a-\frac{t}{2}\right) \int_{0}^{a} x^{*}(s) d s-\frac{a^{2}}{2} \int_{0}^{t} x^{*}(s) d s
$$




$$
\begin{aligned}
& =\int_{0}^{t}(a-s) d s \int_{0}^{a} x^{*}(s) d s-\int_{0}^{a}(a-s) d s \int_{0}^{t} x^{*}(s) d s \\
& =\int_{0}^{t}(a-s) d s \int_{t}^{a} x^{*}(s) d s+\left[\int_{0}^{t}(a-s) d s-\int_{0}^{a}(a-s) d s\right] \int_{0}^{t} x^{*}(s) d s \\
& =\int_{0}^{t}(a-s) d s \int_{t}^{a} x^{*}(s) d s-\int_{t}^{a}(a-s) d s \int_{0}^{t} x^{*}(s) d s \\
& =\int_{0}^{t} \int_{t}^{a}\left[(a-s) x^{*}(u)-(a-u) x^{*}(s)\right] d u d s .
\end{aligned}
$$

But for $0 \leq s \leq t<a$ we have $u=\frac{a-u}{a-s} s+\left(1-\frac{a-u}{a-s}\right) a$ and by the concavity of $x^{*}, x^{*}(u) \geq \frac{a-u}{a-s} x^{*}(s)+\left(1-\frac{a-u}{a-s}\right) x^{*}(a) \geq \frac{a-u}{a-s} x^{*}(s)$. Hence the above integrand - and so the integral - is positive. This proves (4.2).

To prove (4.3), let $s \varepsilon I$ and define

$$
x_{1}(s)=2 a^{-2} x_{0}^{*}(s) \int_{0}^{a} x(u) d u=2 a^{-2}(a-s) \int_{0}^{a} x(u) d u
$$

(recall that $x_{0}(s)=s$ ). Then $(4.2)$ takes the form

$$
\int_{0}^{t} x^{*}(s) d s \leq \int_{0}^{t} x_{1}(s) d s
$$

for all $t \varepsilon I$, with equality at $t=a$. Now the majorant property of $X$ implies that

$$
\|x\|_{X} \leq\left\|x_{1}\right\|_{X}=2 a^{-1} C(X) \int_{0}^{a} x(s) d s,
$$

which is (4.3). This completes the proof of the lemma.

Note that if $X=L_{p}, 1 \leq p \leq \infty$, then $C(X)$ of $(4.3)$ is $C\left(L_{p}\right)=$ $a^{1 / p}(p+1)^{-1 / p}, 1 \leq p<\infty$ and $C\left(L_{\infty}\right)=1$.

Using LEMMA 4.2 we now give two proofs of Grüss' THEOREM 4.1.

First proof. Let

$$
\bar{x}=\frac{1}{a} \int_{0}^{a} x(s) d s \quad \text { and } \quad \bar{y}=\frac{1}{a} \int_{0}^{a} y(s) d s .
$$

Then

$$
\begin{aligned}
D(x, y): & =\frac{1}{a} \int_{0}^{a} x(s) y(s) d s-\frac{1}{a^{2}} \int_{0}^{a} x(s) d s \int_{0}^{a} y(s) d s \\
& =\frac{1}{a} \int_{0}^{a}[x(s)-\bar{x}][y(s)-\bar{y}] d s
\end{aligned}
$$


and by Schwarz's inequality $D(x, y)^{2} \leq D(x, x) D(y, y)$. Now by (4.3) with
$X=L_{2}(I)$,

so that

$$
\int_{0}^{a} x(s)^{2} d s \leq \frac{4}{3} a^{-1}\left(\int_{0}^{a} x(s) d s\right)^{2}
$$

$$
D(x, x) \leq \frac{4}{3} a^{-2}\left(\int_{0}^{a} x(s) d s\right)^{2}-a^{-2}\left(\int_{0}^{a} x(s) d s\right)^{2}=\frac{a^{-2}}{3}\left(\int_{0}^{a} x(s) d s\right)^{2}
$$

and similarly

$$
D(y, y) \leq \frac{a^{-2}}{3}\left(\int_{0}^{a} y(s) d s\right)^{2}
$$

Therefore $D(x, y)^{2} \leq \frac{a^{-4}}{9}\|x\|_{1}^{2}\|y\|_{1}^{2}$ or $|D(x, y)| \leq \frac{a^{-2}}{3}\|x\|_{1}\|y\|_{1}$. But this means that $-\frac{1}{3} a^{-2}\|x\|_{1}\|y\|_{1} \leq D(x, y) \leq \frac{a^{-2}}{3}\|x\|_{1}\|y\|_{1}$ or equivalently

$$
\begin{aligned}
& \int_{0}^{a} x(s) d s \int_{0}^{a} y(s) d s \\
& \quad \leq \frac{3 a}{2} \int_{0}^{a} x(s) y(s) d s \leq 2 \int_{0}^{a} x(s) d s \int_{0}^{a} y(s) d s
\end{aligned}
$$

which in particular proves (4.1).

The second proof of THEOREM 4.1 follows along the lines of that of Barnes [4]. Let

then from (4.2) of LEMMA 4.2

$$
x_{1}(s)=2 a^{-2} s \int_{0}^{a} x(t) d t, y_{1}(s)=2 a^{-2} s \int_{0}^{a} y(t) d t
$$

$$
\begin{aligned}
\int_{0}^{t} x^{*}(s) d s & \leq \frac{t}{a}\left(2-\frac{t}{a}\right) \int_{0}^{a} x^{*}(s) d s=\left(\int_{0}^{a} x(s) d s\right) \int_{0}^{t} 2 a^{-2}(a-s) d s \\
& =\int_{0}^{a} x_{1}^{*}(s) d s
\end{aligned}
$$

and similarly, $\int_{0}^{t} y^{*}(s) d s \leq \int_{0}^{t} y_{1}^{*}(s) d s$ for all $0 \leq t \leq a$, with equality at $t=a$.
Moreover, since $y^{*}$ is concave,

$$
\begin{aligned}
\int_{0}^{t} y_{1}(s) d s & =a^{-2} t^{2} \int_{0}^{a} y(s) d s=a^{-2} t^{2} \int_{0}^{a} y^{*}(s) d s=\frac{t}{a} \int_{0}^{t} y^{*}\left(\frac{a}{t} s\right) d s \\
& \leq \frac{t}{a} \int_{0}^{t} y^{*}\left(\frac{a}{t} s\right) d s+\left(1-\frac{t}{a}\right) y^{*}(a) \int_{0}^{t} d s \\
& \leq \int_{0}^{t} y^{*}\left(s+\left(1-\frac{t}{a}\right) a\right) d s=\int_{0}^{t} y^{*}(a-s) d s
\end{aligned}
$$


Then from the observation following the proof of LEMMA 2.7 and the inequality of Hardy, Littlewood and Pólya [16, Theorem 378]:

$$
\int_{0}^{a} x^{*}(s) y^{*}(a-s) d s \leq \int_{0}^{a} x(s) y(s) d s \leq \int_{0}^{a} x^{*}(s) y^{*}(s) d s
$$

we obtain

$$
\begin{aligned}
\frac{2}{3} a^{-1} & \int_{0}^{a} x(s) d s \int_{0}^{a} y(s) d s \\
& =4 a^{-4} \int_{0}^{a} s(a-s) d s \int_{0}^{a} x(s) d s \int_{0}^{a} y(s) d s \\
& =\int_{0}^{a} x_{1}^{*}(s) y_{1}(s) d s \leq \int_{0}^{a} x^{*}(s) y_{1}(s) d s \leq \int_{0}^{a} x^{*}(s) y^{*}(a-s) d s \\
& \leq \int_{0}^{a} x(s) y(s) d s \leq \int_{0}^{a} x^{*}(s) y^{*}(s) d s \\
& \leq \int_{0}^{a} x_{1}^{*}(s) y^{*}(s) d s \leq \int_{0}^{a} x_{1}^{*}(s) y_{1}^{*}(s) d s \\
& =4 a^{-4} \int_{0}^{a}(a-s)^{2} d s \int_{0}^{a} x(s) d s \int_{0}^{a} y(s) d s \\
& =\frac{4}{3} a^{-1} \int_{0}^{a} x(s) d s \int_{0}^{a} y(s) d s .
\end{aligned}
$$

Again this proves (4.4) and hence THEOREM 4.1.

From THEOREM 4.1 and LEMMA 4.2 we obtain at once the following: If $x, y \varepsilon X$ are positive concave functions on $I$, such that $x y \varepsilon X$, then

$$
\|x\|_{X}\|y\|_{X} \leq 6 \frac{C(X)^{2}}{\varphi_{X}(a)}\|x y\|_{X}
$$

Corollary 4.4 Let $X$ and $Y$ be symmetric spaces on $I$ with the majorant property. Suppose $x \varepsilon X, y \varepsilon Y$ are positive concave functions on $I$. Then

$$
\|x\|_{X}\|y\|_{Y} \leq 6 C(X) C(Y) \frac{1}{a} \int_{0}^{a} x(s) y(s) d s .
$$

Remark 4.5 i) If $X=L_{p}, 1 \leq p \leq \infty$, inequality (4.3) takes the form

$$
\frac{(p+1)^{1 / p}}{2} a^{1-1 / p}\|x\|_{p} \leq \int_{0}^{a} x(s) d s .
$$


For $p=2$ this inequality is due to Frank-Pick (1915) and for general $p$ to
Favard (1933).

ii) If $X=L_{p}, Y=L_{q}, 1 \leq p, q \leq \infty$, inequality (4.7) takes the form

$$
\frac{1}{6}(p+1)^{1 / p}(q+1)^{1 / q} a^{1-1 / p-1 / q}\|x\|_{p}\|y\|_{q} \leq \int_{0}^{a} x(s) y(s) d s
$$

which was proved by Barnes [4]. Generalizations of (4.8) and (4.9) to a class larger than concave functions and to the multidimensional case were given
by Borell ([7] [8]).

\section{On the Ky Fan inequality and some gen- eralizations.}

Let $K: I \times I \rightarrow[0, \infty)$ be a function satisfying

$$
\begin{aligned}
& \int_{0}^{a} K(s, t) d s \leq B w(t) \\
& \int_{0}^{a} K(s, t) d t \leq B w(s)
\end{aligned}
$$

where $B$ is a positive constant and $w$ a positive integrable function.

If $K=C$ a constant, then (5.1) holds with $B=C a$ and $w \equiv 1$. Also, if $K(s, t)=k(s) \ell(t)$, where $k$ and $\ell$ are positive integrable functions on $I$, then

(5.1) is satisfied with $B=\max \left(\|k\|_{1},\|\ell\|_{1}\right)$ and $w(t)=\max (k(t), \ell(t))$.
$\mathrm{Ky}$ Fan [13] proved the following generalization of Chebyshev's inequality
for $w \equiv 1$. for $w \equiv 1$.

Theorem 5.1 Suppose $K$ satisfies (5.1). If $x$ and $y$ are positive decreasing

$$
\int_{0}^{a} \int_{0}^{a} K(s, t) x(s) y(t) d s d t \leq B \int_{0}^{a} w(s) x(s) y(s) d s .
$$

Proof (cf. [28]). Observe that (5.2) is linear in $x$. Since $x$ is decreasing it can be approximated by a sum of a finite number of step functions that have a single jump. It suffices therefore to establish (5.2) in the special case 
where $x(s)=1$ if $0 \leq s \leq c$ and 0 if $c<s \leq a$. For this $x,(5.2)$ takes the form

$$
\int_{0}^{a} \int_{0}^{c} K(s, t) y(t) d s d t \leq B \int_{0}^{c} w(s) y(s) d s .
$$

But to prove (5.3) it suffices again to consider $y$ to be a single jump function, say $y(s)=1$ if $0 \leq s \leq d$ and 0 if $d<s \leq a$. Then there are two cases to consider, namely $c \leq d$ and $d \leq c$. In the first case (5.3) becomes

$$
\int_{0}^{d} \int_{0}^{c} K(s, t) d s d t \leq B \int_{0}^{c} w(s) d s
$$

which by (5.1) is clearly valid. The second case follows from the first by interchanging $x$ and $y$. This proves the theorem.

The next result is a generalization but with a different constant - namely 2. We show this constant to be sharp.

Theorem 5.2 Suppose $K$ satisfies (5.1). If $f$ is a measurable function such that $f: I \times I \rightarrow[0, \infty)$ decreases in each variable separately, then

$$
\int_{0}^{a} \int_{0}^{a} K(s, t) f(s, t) d s d t \leq 2 B \int_{0}^{a} w(s) f(s, s) d s .
$$

Moreover, the constant 2 in (5.4) is sharp.

Proof. For $s, t \varepsilon I$, we have

$$
\begin{aligned}
f(s, t) & =f(s, t) 1_{[0, t]}(s)+f(s, t) 1_{[t, a]}(s) \\
\leq & f(s, s) 1_{[0, t]}(s)+f(t, t) 1_{[t, a]}(s) \\
\leq & f(s, s)+f(t, t), \text { so that } \\
\int_{0}^{a} \int_{0}^{a} K(s, t) f(s, t) d s d t \leq & \int_{0}^{a} \int_{0}^{a} K(s, t) f(s, s) d s d t \\
& \quad+\int_{0}^{a} \int_{0}^{a} K(s, t) f(t, t) d s d t \\
& \leq B \int_{0}^{a} w(s) f(s, s) d s+B \int_{0}^{a} w(t) f(t, t) d t \\
& =2 B \int_{0}^{a} w(s) f(s, s) d s .
\end{aligned}
$$


To show that the constant 2 is sharp, let $a=1$ and $K(s, t)=w(s) w(t)$, $w$ positive and integrable on $[0,1]$. For $0<\alpha<1$, define $f(s, t)=n$ if $0 \leq s \leq \alpha$ or $0 \leq t \leq \alpha$, and equal to 1 otherwise. Then

$$
\begin{array}{r}
L(\alpha, n):=\int_{0}^{1} \int_{0}^{1} K(s, t) f(s, t) d s d t=n \int_{0}^{\alpha} w(s) d s \int_{0}^{1} w(t) d t \\
+n \int_{\alpha}^{1} w(s) d s \int_{0}^{\alpha} w(t) d t+\int_{\alpha}^{1} w(s) d s \int_{\alpha}^{1} w(t) d t
\end{array}
$$

and since $B=\int_{0}^{1} w(s) d s$ (in this case) it follows that

$$
P(\alpha, n):=B \int_{0}^{1} f(s, s) w(s) d s=\int_{0}^{1} w(s) d s\left[n \int_{0}^{\alpha} w(s) d s+\int_{\alpha}^{1} w(s) d s\right] .
$$
implies the constant is sharp.

Since the case $f(s, t)=x(s) y(t)$ in (5.4) is known to give a better constant than 2 (cf. THEOREM 5.1), it is natural to search for those classes of functions $f(s, t)$ for which the constant in (5.4) is 1 . We shall see below that the positive set functions represent a large class of functions with this
property.

Definition 5.3 A function $f: I \times I \rightarrow[0, \infty)$ is said to be a positive set function (P.S.F.) if

$$
\begin{array}{r}
\triangle_{h, k}^{2} f(s, t):=f(s+h, t+k)-f(s+h, t) \\
-f(s, t+k)+f(s, t) \geq 0
\end{array}
$$

holds for all $h, k \geq 0$ (or $h, k \leq 0$ ) with $s+h, t+k \varepsilon I$, and for arbitrary
choices of $s, t \varepsilon I$.

Of course, if $f$ is smooth, i.e. $f$ has continuous second partial derivatives, then (5.5) is equivalent to $\frac{\partial^{2} f}{\partial s \partial t}(s, t) \geq 0$.

If $f(s, t)=x(s)+C y(s)$, where $x$ and $y$ are positive on $I$ and $C$ a real constant then $\Delta_{h, k}^{2} f(s, t)=0$ so that $f$ is a P.S.F. Similarly, if $f(s, t)=$ $x(s) y(t)$, where $x$ and $y$ are either both increasing or decreasing, then

$$
\triangle_{h, k}^{2} f(s, t)=[x(s+h)-x(s)][y(t+k)-y(t)]
$$


shows that $f$ is a P.S.F. Another example is the following: Let $\varphi$ be a convex increasing on $[0, \infty)$ and $f$ a P.S.F. If either $f$ decreases in each variable separately or increases in each variable separately, then $F$ defined by $F(s, t)=\varphi(f(s, t))$ is a P.S.F. To see this, suppose $f$ increases in each variable separately and $h, k>0$. Let

$$
x_{1}=f(s, t)<x_{2}=f(s+h, t), \bar{x}_{2}=f(s, t+k)<x_{3}=f(s+h, t+k),
$$

then by the convexity of $\varphi$

$$
\frac{\varphi\left(x_{2}\right)-\varphi\left(x_{1}\right)}{x_{2}-x_{1}} \leq \frac{\varphi\left(x_{3}\right)-\varphi\left(x_{1}\right)}{x_{3}-x_{1}} \leq \frac{\varphi\left(x_{3}\right)-\varphi\left(\bar{x}_{2}\right)}{x_{3}-\bar{x}_{2}}
$$

Hence

$$
\begin{aligned}
\triangle_{h, k}^{2} F(s, t) & =\varphi\left(x_{3}\right)-\varphi\left(\bar{x}_{2}\right)-\varphi\left(x_{2}\right)+\varphi\left(x_{1}\right) \\
& \geq \frac{x_{3}-\bar{x}_{2}}{x_{2}-x_{1}}\left[\varphi\left(x_{2}\right)-\varphi\left(x_{1}\right)\right]-\varphi\left(x_{2}\right)+\varphi\left(x_{1}\right) \\
& =\frac{x_{3}-\bar{x}_{2}-x_{2}+x_{1}}{x_{2}-x_{1}}\left[\varphi\left(x_{2}\right)-\varphi\left(x_{1}\right)\right]
\end{aligned}
$$

and since $f$ is a P.S.F. and $\varphi$ is increasing, it follows that $\triangle_{h, k}^{2} F(s, s t) \geq 0$.

We conclude with the following main result:

Theorem 5.4 Let $K$ be a positive continuous function on $I \times I$ and suppose $f: I \times I \rightarrow[0, \infty)$ is a continuous P.S.F.

a) If for all teI

$$
\int_{t}^{a} \int_{0}^{t} K(u, v) d v d u=\int_{0}^{t} \int_{t}^{a} K(u, v) d v d u
$$

then

$$
\int_{0}^{a} \int_{0}^{a} K(s, t) f(s, t) d s d t \leq \int_{0}^{a}\left(\int_{0}^{a} K(s, t) d s\right) f(t, t) d t
$$

b) If for all $s, t \varepsilon I$

$$
\int_{0}^{a} K(u, t) d u=B w(t) \quad \text { and } \quad \int_{0}^{a} K(s, v) d v=B w(s)
$$

then

$$
\int_{0}^{a} \int_{0}^{a} K(s, t) f(s, t) d s d t \leq B \int_{0}^{a} w(s) f(s, s) d s
$$


Proof. The method of proof is taken from Pečaric [22]. We prove the result first for smooth functions and then via Bernstein polynomials and the Weierstrass approximation theorem the general result. First some notations:
Write

$$
\begin{aligned}
I(f) & =\int_{0}^{a} \int_{0}^{a} K(s, t) f(s, t) d s d t \quad I_{0}(f)=\int_{0}^{a} w(s) f(s, s) d s \\
J(f) & =\int_{0}^{a} \int_{0}^{a} K(s, t)[f(t, t)-f(s, t)] d t d s \\
P(s, t) & =\int_{0}^{t} K(s, v) d v, \quad Q(s, t)=\int_{t}^{a} K(s, v) d v \\
\bar{Q}(s, t) & =\int_{s}^{a} K(u, t) d u \\
W(t) & =\int_{t}^{a} w(s) d s, \quad U(s, t)=\int_{s}^{a} \int_{0}^{t} K(u, v) d v d u \\
\bar{U}(s, t) & =\int_{0}^{s} \int_{t}^{a} K(u, v) d v d u \\
V(s, t) & =\int_{s}^{a} \int_{t}^{a} K(u, v) d v d u
\end{aligned}
$$

Clearly, $P(s, 0)=Q(s, a)=\bar{Q}(a, t)=U(a, t)=\bar{U}(0, t)=0$.

Step I. Assume $f_{1}=\frac{\partial f}{\partial s}, f_{2}=\frac{\partial f}{\partial t}$ and $f_{21}=\frac{\partial^{2} f}{\partial s \partial t}$ exist and are continuous. Since

a) If we show that $J(f) \geq 0$ part a) follows under these assumptions.

$$
\begin{gathered}
\int_{0}^{a} K(s, t)[f(t, t)-f(s, t)] d t=\left(\int_{0}^{s}+\int_{0}^{a}\right) K(s, t)[f(t, t)-f(s, t)] d t \\
=\int_{0}^{s} \frac{\partial P}{\partial t}(s, t)[f(t, t)-f(s, t)] d t-\int_{s}^{a} \frac{\partial Q}{\partial t}(s, t)[f(t, t)-f(s, t)] d t \\
=-\int_{0}^{s} P(s, t)\left[f_{1}(t, t)+f_{2}(t, t)-f_{2}(s, t)\right] d t \\
\quad+\int_{s}^{a} Q(s, t)\left[f_{1}(t, t)+f_{2}(t, t)-f_{2}(s, t)\right] d t
\end{gathered}
$$

we obtain

$$
\begin{aligned}
J(f)= & -\int_{0}^{a} \int_{0}^{s} P(s, t)\left[f_{1}(t, t)+f_{2}(t, t)-f_{2}(s, t)\right] d t d s \\
& +\int_{0}^{a} \int_{s}^{a} Q(s, t)\left[f_{1}(t, t)+f_{2}(t, t)-f_{2}(s, t)\right] d t d s
\end{aligned}
$$




$$
\begin{aligned}
= & -\int_{0}^{a} \int_{t}^{a} P(s, t)\left[f_{1}(t, t)+f_{2}(t, t)-f_{2}(s, t)\right] d s d t \\
& +\int_{0}^{a} \int_{0}^{t} Q(s, t)\left[f_{1}(t, t)+f_{2}(t, t)-f_{2}(s, t)\right] d s d t \\
= & -\int_{0}^{a} U(t, t) f_{1}(t, t) d t-\int_{0}^{a} U(t, t) f_{2}(t, t) d t \\
& +\int_{0}^{a} \int_{t}^{a} P(s, t) f_{2}(s, t) d s d t+\int_{0}^{a} \bar{U}(t, t) f_{1}(t, t) d t \\
& +\int_{0}^{a} \bar{U}(t, t) f_{2}(t, t) d t-\int_{0}^{a} \int_{0}^{t} Q(s, t) f_{2}(s, t) d s d t .
\end{aligned}
$$

However,

$$
\begin{aligned}
\int_{t}^{a} P(s, t) f_{2}(s, t) d s & =-\int_{t}^{a} \frac{\partial U}{\partial s}(s, t) f_{2}(s, t) d s \\
& =U(t, t) f_{2}(t, t)+\int_{t}^{a} U(s, t) f_{21}(s, t) d s
\end{aligned}
$$

and similarly

$$
\begin{aligned}
-\int_{0}^{t} Q(s, t) f_{2}(s, t) d s & =-\int_{0}^{t} \frac{\partial \bar{U}}{\partial s}(s, t) f_{2}(s, t) d s \\
& =-\bar{U}(t, t) f_{2}(t, t)+\int_{0}^{t} \bar{U}(s, t) f_{21}(s, t) d s
\end{aligned}
$$

Substituting, we obtain

$$
\begin{array}{rl}
J(f)=-\int_{0}^{a} & U(t, t) f_{1}(t, t) d t+\int_{0}^{a} \int_{t}^{a} U(s, t) f_{21}(s, t) d s d t \\
& +\int_{0}^{a} \vec{U}(t, t) f_{1}(t, t) d t+\int_{0}^{a} \int_{0}^{t} \vec{U}(s, t) f_{21}(s, t) d s d t .
\end{array}
$$
But since $U(t, t)=\bar{U}(t, t)$, it follows that by the mean value theorem, for
some $\xi, \eta \varepsilon I$

$$
J(f)=\int_{0}^{a} \int_{0}^{a} \tilde{U}(s, t) f_{21}(s, t) d s d t=f_{21}(\xi, \eta) \int_{0}^{a} \int_{0}^{a} \tilde{U}(s, t) d s d t
$$

where $\tilde{U}(s, t)=U(s, t)$ if $t \leq s \leq a$ and $\bar{U}(s, t)$ if $0 \leq s \leq t$. But since the integrand is positive $f_{21}(\xi, \eta) \geq 0$ we obtain $J(f) \geq 0$. 
b) We must show that $B I_{0}(f)-I(f) \geq 0$ in this case. Since

it follows that

$$
\begin{aligned}
\int_{0}^{a} K(s, t) f(s, t) d t & =-\int_{0}^{a} \frac{\partial Q}{\partial t}(s, t) f(s, t) d t \\
& =Q(s, 0) f(s, 0)+\int_{0}^{a} Q(s, t) f_{2}(s, t) d t
\end{aligned}
$$

$$
\begin{aligned}
I(f)= & \int_{0}^{a} Q(s, 0) f(s, 0) d s+\int_{0}^{a} \int_{0}^{a} Q(s, t) f_{2}(s, t) d s d t \\
= & -\int_{0}^{a} \int_{0}^{a} \frac{\partial \bar{Q}}{\partial s}(s, v) f(s, 0) d s d v-\int_{0}^{a} \int_{0}^{a} \frac{\partial V}{\partial s}(s, t) f_{2}(s, t) d s d t \\
= & \int_{0}^{a}\left[\bar{Q}(0, v) f(0,0)+\int_{0}^{a} \bar{Q}(s, v) f_{1}(s, 0) d s\right] d v \\
& \quad \quad \quad \int_{0}^{a}\left[V(0, t) f_{2}(0, t)+\int_{0}^{a} V(s, t) f_{21}(s, t) d s\right] d t \\
= & f(0,0) V(0,0)+\int_{0}^{a} V(s, 0) f_{1}(s, 0) d s \\
& \quad+\int_{0}^{a} V(0, t) f_{2}(0, t) d t+\int_{0}^{a} \int_{0}^{a} V(s, t) f_{12}(s, t) d s d t .
\end{aligned}
$$

Also

But since

$$
\begin{aligned}
I_{0}(f) & =\int_{0}^{a} w(s) f(s, s) d s=-\int_{0}^{a} \frac{\partial W(s)}{\partial s} f(s, s) d s \\
& =W(0) f(0,0)+\int_{0}^{a} W(s)\left[f_{1}(s, s)+f_{2}(s, s)\right] d s .
\end{aligned}
$$

and similarly (since $f_{12}=f_{21}$ )

$$
\begin{aligned}
\int_{0}^{a} \int_{0}^{a} W(t) f_{21}(s, t) d t d s & =\int_{0}^{a} \int_{0}^{t} W(t) f_{21}(s, t) d s d t \\
& =\int_{0}^{a} W(t) f_{2}(t, t) d t-\int_{0}^{a} W(t) f_{2}(0, t) d t
\end{aligned}
$$

$$
\int_{0}^{a} \int_{0}^{s} W(s) f_{21}(s, t) d t d s=\int_{0}^{a} W(s) f_{1}(s, s) d s-\int_{0}^{a} W(s) f_{1}(s, 0) d s,
$$

it follows on substituting that

$$
\begin{gathered}
I_{0}(f)=W(0) f(0,0)+\int_{0}^{a} W(s) f_{1}(s, 0) d s+\int_{0}^{a} W(t) f_{2}(0, t) d t \\
+\int_{0}^{a} \int_{0}^{a} W(\max (s, t)) f_{21}(s, t) d t d s .
\end{gathered}
$$


Therefore

$$
\begin{aligned}
B I_{0}(f)-I(f)=f(0,0) & {[B W(0)-V(0,0)]+\int_{0}^{a}[B W(s)-V(s, 0)] f_{1}(s, 0) d s } \\
& +\int_{0}^{a}[B W(t)-V(0, t)] f_{2}(0, t) d t \\
& +\int_{0}^{a} \int_{0}^{a}[B W(\max (s, t))-V(s, t)] f_{21}(s, t) d s d t \geq 0 .
\end{aligned}
$$

This proves b) under the smoothness assumptions on $f$.

Step II. If $f$ is an arbitrary continuous P.S.F., then we now approximate $f$ uniformly on $I \times I$ by polynomials which are (smooth) P.S.F.

a) It is known that the Bernstein polynomials

$$
\begin{aligned}
P(s, t): & =P_{m, n}(s, t) \\
& =\sum_{i=0}^{m} \sum_{j=0}^{n} a_{i j}\left(\begin{array}{c}
m \\
j
\end{array}\right)\left(\begin{array}{c}
n \\
j
\end{array}\right)(a-s)^{m-i} s^{i}(a-t)^{n-j} t^{j}
\end{aligned}
$$

with $a_{i j}=f\left(\frac{i}{m} a, \frac{j}{n} a\right)$ converge uniformly to $f$ on $I \times I$ as $m, n \rightarrow \infty$. Further

$$
\begin{aligned}
\frac{\partial P}{\partial t}= & n \sum_{j=0}^{n-1}\left(\begin{array}{c}
n-1 \\
j
\end{array}\right)(a-t)^{n-1-j} t^{j} \\
& \cdot \sum_{i=0}^{m-1}\left(a_{i, j+1}-a_{i, j}\right)\left(\begin{array}{c}
m \\
i
\end{array}\right)(a-s)^{m-i} s^{i}
\end{aligned}
$$

and

$$
\frac{\partial^{2} P}{\partial s \partial t}=m n \sum_{i=0}^{m-1} \sum_{j=0}^{n-1}\left(\begin{array}{c}
m-1 \\
i
\end{array}\right)\left(\begin{array}{c}
n-1 \\
j
\end{array}\right) A_{i j}(a-s)^{m-1-i} s^{i}(a-t)^{n-1-j} t^{j},
$$

where $A_{i j}=a_{i+1, j+1}-a_{i+1, j}-a_{i, j+1}+a_{i, j}$. But since $f$ is a P.S.F., $A_{i j} \geq 0$, and so $\frac{\partial^{2} P}{\partial s \partial t} \geq 0$ on $I \times I$. Therefore, $P$ is a P.S.F. which satisfies the conditions on Step I. By what we have proved, $J(P) \geq 0$ and so by the Weierstrass approximation theorem

$$
\begin{aligned}
0 & \leq J(P)=J(P-f)+J(f) \\
& \leq 2 \sup _{s, t e I}|P(s, t)-f(s, t)| \int_{0}^{a} \int_{0}^{a} K(s, t) d s d t+J(f)<2 \varepsilon U(0, a)+J(f) .
\end{aligned}
$$


But $\varepsilon>0$ is arbitrary, so that $J(f) \geq 0$. Since

b) This part follows also from the Weierstrass approximation theorem:

$$
0 \leq B I_{0}(P)-I(P)=B I_{0}(P-f)-I(P-f)+B I_{0}(f)-I(f),
$$

and $B I_{0}(P-f)$ and $I(P-f)$ may be choosen as small as we please the result follows.

Remark 5.5 If $f$ is as in THEOREM 5.4 and $K(s, t)=w(s) w(t)$, where $w$ is positive and integrable on $I$, then (5.6) and (5.7) have the form

$$
\int_{0}^{a} w(t) \int_{0}^{a} f(s, t) w(s) d s d t \leq \int_{0}^{a} w(t) d t \int_{0}^{a} f(s, s) w(s) d s .
$$

\section{P.L. Chebyshev - his life and mathemat- ical work in short}

Pafnutii L'vovich Chebyshev, the son of an old Russian noble family, was born on May 16 (May 4, old style) 1821 in the village of Okatovo, district Borovsk, government Kaluga in Russia. After primary school at home and secondary school in Moscow, he studied at the University of Moscow - primarily under N.D. Brashman (1796-1866). He completed his Magister-thesis on an "Elementary analysis of probability theory" in 1846 and moved to the University of St. Petersburg (Leningrad) in 1847 where he studied number theory under V.Ya. Bunyakovskili (1804-1889). In 1849 he completed his D.Sc. Thése on "Theory of congruences" and in 1853 became Extraordinary Professor and in 1857 Ordinary Professor at the University of St. Petersburg. He never married.

Chebyshev's merits were recognized early in his career. He was elected a Corresponding Member of the Société Royale des Sciences de Liège and of the Société Philomathique in 1856, of the Paris Academy of Sciences in 1860 and a Foreign Member in 1874 (the first Russian since Peter the Great), as well as a corresponding or foreign member of the Berlin Academy of Sciences (1871), the Bologna Academy (1973), the Royal Society of London (1877), the Italian Royal Academy (1880), and the Swedish Academy of Sciences 
(1893). He was also a member of the St. Petersburg Academy of Sciences (1856, ordinary member 1859).

His scholarly work consisted of over 80 research papers and covered a wide range of subjects including number theory, probability theory, quadratic forms, orthogonal functions and polynomials, theory of integrals, approximation theory, bearings, the construction of geographic maps and he derived various formulas for the computation of volumes. He studied also theoretical mechanics and devoted much attention to the problem of obtaining rectilinear motion from rotary motion by mechanical linkage. The "Chebyshev parallel motion" is a three-bar linkage that gives a very good approximation to exact rectilinear motion. He created over 40 new mechanisms and improved over 80. Many of these were demonstrated at the 1878 and 1883 exhibitions in Paris and Chicago. His name is associated with many mathematical concepts and areas, for example:

- in the number theory he studied the so called Chebyshev function $\Pi(x)$, i.e., the number of primes below a given number $x$ and proved that $\Pi(x) \cong$ $x / \log x$ as $x \rightarrow \infty, 0,92129<\Pi(x) \log x / x<1,10555$; also he proved the Bertrand postulate - for $n>3$ there is at least one prime number in the interval $(n, 2 n-2)$.

- he developed a basic inequality of probability theory called Chebyshev inequality, a generalized form of the Bienaymé-Chebyshev inequality (Bienaymé 1853 , Chebyshev 1866), and used the latter inequality to give a very simple and precise proof of the Chebyshev theorem (generalized law of large numbers).

- he introduced and investigated the Chebyshev polynomials (1854)

$$
T_{n}(x)=\cos (n \arccos x) \text { for } n=0,1,2, \ldots
$$

and

$$
U_{n}(x)=\sin (n \arcsin x) \text { for } n=1,2,3, \ldots
$$

- Chebyshev theorem (1953) on the integration of binomial differentials $\int x^{m}\left(a+b x^{n}\right)^{p} d x$, where $a, b$ are real numbers and $m, n$ and $p$ are rational numbers, cannot be expressed in terms of elementary functions for any $m, n$ and $p$, except in the case where (at least) one of $p,(m+1) / n$ and $p+(m+1) / n$ is an integer.

- Chebyshev approximation (1853) is a problem of best uniform approximation of a continuous function by algebraic polynomials of degree not exceeding $n$. 
- Chebyshev inequality (1882), which we discussed in Section 2. Moreover, well known are the Chebyshev set, Chebyshev alternation theorem, Chebyshev iteration method (1881), Chebyshev root-finding method, Chebyshev quadrature formula (1874), Chebyshev system, Chebyshev constant, Chebyshev centre, Chebyshev net (1878), Chebyshev node, Chebyshev differential equation,
etc.

He was the founder of the St. Petersburg Mathematical School (called also Chebyshev School) and had such illustrious students as: A.N. Korkin (1837-1908), E.I. Zolotarev (1847-1878), A.V. Vassiliev (1853-1929), A.A. Markov (1856-1922), A.M. Lyapunov (1857- 1918), C.A. Posse (1847-1928), D.A. Grave (1863-1939). V.A. Steklov (1864-1926), G.F. Voronoi (18681908), and A.N. Krylov (1863-1945).

His mathematical work contributed significantly to the powerful mathematical tradition which we find in the Soviet Union today.

Chebyshev retired from the university in 1882, and at the age of 73 died from paralysis of the heart in St. Petersburg on December 8 (November 26) 1894. A selection of Chebyshev's research was published in two volumes by Markoff and Sonin [19] and was translated into French in 1962. In 1946-51 the Soviet Academy of Sciences edited in five volumes his Complete Collected $[19],[9]$ and to the references given in [9].

\section{Acknowledgement}

The authors wish to thank the referee for a number of comments and suggestions which led to an improvement of the paper.

\section{$\underline{\text { References }}$} i-j Anucmiya, A generalization of Riesz-Fischer's theorem, J. Math. Soc.
Japan 5(1953), 353-354.

[2] B.J. Andersson, An inequality for convex functions, Nord. Math. Tidskr. 6(1958), 25-26.

[3] K.A. Andreiev, Note sur une relation entre les intégrales définies des produits des functions, Mém. Soc. Ci. Bordeaux 32 (1883), 1-14. 
[4] D.C. Barnes, Some complements of Hölder's inequality, J. Math. Anal. Appl 26(1969), 82-87.

[5] P.R. Beesack and J.E. Pečarič, Integral inequalities of Čebyšev type, J. Math. Anal. Appl. 111(1985), 643-659.

[6] C. Bennett and R. Sharpley, Interpolation of Operators, Pure and Applied Math. 129, Academic Press, Boston 1988.

[7] C. Borell, Inverse Hölder inequalities in one and several dimensions, J. Math. Anal. Appl. 41(1973), 300-312.

[8] C. Borell, Integral inequalities for generalized concave and convex functions. J. Math. Anal. Appl. 43(1973), 419-440.

[9] P.L. Butzer and F. Jongmans, P.L. Chebyshev (1821-1894) and his contacts with western European scientists, Historia Math. 16 (1989), 46-68.

[10] P.L. Chebyshev, On approximating expressions of one integrals by another, Proc. Math. Soc. Kharkov 2(1882), 93-98 (Russian); In: Complete Collected Works, Vol. III (1948), 128-131 and Oeuvres, Vol. II (1907), 716-719.

[11] P.L. Chebyshev, Complete Collected Works, Izdatel'stvo A kademii Nauk SSR, Moscow-Leningrad: Vol. I, 1946, 342 pp.; Vol. II, 1947, 520 pp.; Vol. III, 1948, 414 pp.; Vol. IV, 1948, 255 pp.; Vol. $V$ : Other Works, Biographical Materials, 1951, 474 pp. (Russian).

[12] P.L. Chebyshev; Oeuvres I,II, Chelsea Publ., N.Y. 1962.

[13] K. Fan, An integral inequality, Amer. Math. Monthly 60(1953), 195197.

[14] A.M. Fink and Max Jodeit, Jr. On Chebyshev's other inequality, Inequalities in Statistics and Probability, IMS Lecture Notes - Monograph Series Vol. 5 (1984) 115-120.

[15] G. Grüss, Über das Maximum des absoluten Betrages von (1/(ba)) $\int_{a}^{b} f(x) g(x) d x-\left(1 /(b-a)^{2}\right) \int_{a}^{b} f(x) d x \int_{a}^{b} g(x) d x$, Math. Z. 39(1935), 215-226. 
[16] G.H. Hardy, J.E. Littlewood and G. Pólya, Inequalities, Cambridge 1934.

[17] S.G. Krein, Yu. I. Petunin and E.M. Semenov, Interpolation of Linear Operators, Providence 1982.

18] G.G. Lorentz, Bernstein Polynomials, Univ. of Toronto Press, Toronto 1953

[19] A. Markoff and N. Sonin (Eds.), Oeuvres de P.L. Chebyshev, St. Petersburg: Vol. I, 1899; Vol. II, 1907.

[20] D.S. Mitrinovič, Analytic Inequalities, Springer-Verlag, Berlin 1970.

[21] D.S. Mitrinovič and P.M. Vasic. History, variations and generalizations of the Cebyšev inequality and the question of some priorities, Univ. Beograd. Publ. Elektrotehn. Fak. Ser. Mat. Fiz. No. 461-497 (1974), 1-30.

[22] J.E. Pečarič, Some further remarks on the Ostrowski generalization of Cebysev's inequality, J. Math. Anal. Appl. 102 (1984), 18-33.

[23] N.A. Sapogov, On an inequality of Chebyshev, Uspekhi Mat. Nauk 6 (1951), 157-159 (Russian).

[24] D.W. Sasser and M.L. Slater, On the inequality $\sum x_{i} y_{i} \geq 1 / n \sum x_{i} \sum y_{i}$ and the van der Waerden permanent conjecture, J. Combinatorial Theory 3 (1967), 25-33.

[25] A.A. Sedaev, The best constant in Chebyshev inequality for symmetric spaces, In: Applicable Methods of Functional Analysis, Voronezh 1985, 127-131 (Russian).

[26] E.M. Semenov, A new interpolation theorem, Funkcional Anal. $i$ Prilozen 2(1968), 68-80; English transl. in Functional Anal. Appl. 2 (1968).

[27] J.F. Steffensen, En Ulighed mellem Middelvaerdier, Mat. Tidskr. 1925B, 49-53.

[28] S.K. Stein, An inequality in two monotonic functions Amer. Math. Monthly 83 (1976), 469-471. 
[29] A.C. Zaanen, Integration, North-Holland, Amsterdam 1967.

Received January 9, 1991 